\title{
Hydrodynamical evaluation of microporous covered stent for the treatment of intracranial aneurysms: Comparison of flow reduction property with flow diverter stent by using particle imaging velocimetry and in vitro flow simulator
}

\author{
Takeshi Moriwaki ${ }^{1,2, *}$, Tsutomu Tajikawa ${ }^{3}$, Yasuhide Nakayama ${ }^{2}$ \\ ${ }^{I}$ Faculty of Science and Technology, Hirosaki University, Aomori, Japan \\ ${ }^{2}$ Department of Artificial Organs, National Cerebral and Cardiovascular Center Research Institute, Osaka, Japan \\ ${ }^{3}$ Faculty of Engineering Science, Kansai University, Osaka, Japan
}

Received: 15 January 2018 / Accepted: 24 May 2018

(C) Japanese Society of Biorheology 2018

\begin{abstract}
We have developed a microporous covered stent (MCS) for the treatment of intracranial aneurysms (IAs). MCS promotes intentional thrombosis in the IA by blood stagnation and facilitates IA embolization treatment by clotting of whole blood in the IA. Flow reduction is a key property of such stent-type IA embolization devices. In this study, we evaluated the flow reduction property of MCS, and compered that of a general balloon expandable stent (bare stent, BS) for supporting a stenotic vessel and a typical flow diverter stent (FD) for IA treatment. This was carried out using particle imaging velocimetry (PIV) and an in vitro flow simulator with a saccular IA and straight parent vessel model. The PIV results show that the area mean shear rates in the IA flow were reduced to $34.7 \%$ $\left(5.56 \mathrm{~s}^{-1}\right)$ for the BS model, 9.2\% $\left(1.48 \mathrm{~s}^{-1}\right)$ for the FD model, and $2.7 \%\left(0.43 \mathrm{~s}^{-1}\right)$ for the MCS model with respect to the unstented condition $\left(16.04 \mathrm{~s}^{-1}\right)$ at a Reynolds number of 600. Considering that the flow reduction property of the MCS model is three times higher than that of the FD model, it is clear that the MCS facilitates more reliable IA embolization than FD.
\end{abstract}

Keywords covered stent, aneurysm, embolization, particle image velocimetry, flow diverter

\section{Introduction}

For the treatment of unruptured intracranial aneurysms

*E-mail: moriwaki@hirosaki-u.ac.jp, TEL: +81-172-39-3690, FAX: +81-172-39-3690
(IAs), clipping [1] and coiling [2] are widely performed. Whereas stent-assisted coiling [3] has been used to treat wide-neck IAs deep in the brain, clipping is difficult as there is a risk of falling coil. Flow diverter devices (FDs) [4-6], fine mesh stents that promote flow reduction and induce thrombosis in IA, have recently been developed and approved for clinical use.

Microporous covered stents (MCSs) [7-9] have also been developed for the treatment of giant and/or wide-neck IAs. An MCS is completely embedded within a thin microporous film of segmented polyurethane, which provides a flat luminal surface. By adjusting the pore size and porosity of the microporous film, flow reduction is controlled to induce thrombosis in IA and to not excessively inhibit blood flow in side-branch vessels [8]. To investigate the MCS's easy-navigation, IA embolization, and side-branch patency preservation properties, we previously performed in vivo studies [9].

The flow reduction property of FD or MCS affects the treatment performance for IA. By shielding the IA neck using a stent, blood flow entering the IA from the parent vessel is significantly reduced. Various studies have demonstrated that thrombus formation is promoted by reducing hydrodynamic parameters, especially shear rate [10, 11]. Stagnation of the blood flow in the IA causes thrombosis of the entire IA area, which enables IA treatment to be accomplished via endothelialization of the stent lumen. Many studies have reported on flow reduction property [12], thrombus formation [13], and the relationship between flow parameter and treatment result [14] using FD. In general, it is known that flow reduction is related to the porosity of stents $[15,16]$. Tajikawa et al. reported that pore diameter significantly influences flow reduction [17]. 
This study was conducted with the objectives of evaluating the flow reduction property of MCS and comparing it to that of other stents. To this end, visualization measurement of IA flow was conducted using an in vitro flow simulator with an IA model and the particle image velocimetry (PIV) technique. More specifically, the flow velocity distribution in the central cross-section of the IA was measured and the shear rate calculated for a saccular aneurysm formed on the sidewall of a straight parent vessel.

\section{Materials and Methods}

\subsection{Stent model}

Three types of stent models were made and their flow reduction property compared. The first was a bare stent (BS) model with an imitated general balloon expandable mesh pattern for supporting a stenotic vessel. The second was an FD model with an imitated mesh pattern of Pipeline Flex [12], a typical flow diverter stent, for IA treatment. The third was an MCS model, with the imitated mesh pattern of our developing stent (NCVC-CS1) [9] for IA treatment. The diameter and length of NCVC-CS1 were 3.5-5.0 $\mathrm{mm}$ and 16-28 $\mathrm{mm}$, respectively. Each stent model was fabricated by laser processing (Femtosecond laser micro-nanomachining system, Tokyo Instrument Inc., wavelength: $800 \mathrm{~nm}$, repetition frequency: $1 \mathrm{kHz}$, pulse energy: $1 \mathrm{~mJ}$, pulse duration: $90 \mathrm{fs}$ ) from a $0.03 \mathrm{~mm}$ thick stainless steel sheet. The overall sheet size was $25 \times 20 \mathrm{~mm}$ and the mesh area was $20 \times 15 \mathrm{~mm}$. The mesh patterns of the fabricated sheets are shown in Fig. 1. Further, the pore size and porosity of the fabricated sheets are presented in Table 1.

\subsection{In vitro flow simulator with IA model}

The experimental setup has already been presented in detail in a previous report [17]. Thus, only a brief outline is given here. A schematic of the flow simulator is shown in
Fig. 2. The working fluid was a mixture of glycerin (53 wt $\%$ ) and water (47 wt $\%$ ) with density $1130 \mathrm{~kg} / \mathrm{m}^{3}$ and kinematic viscosity $v=4.4 \times 10^{-6} \mathrm{~m}^{2} / \mathrm{s}$. A steady flow was generated by a centrifugal pump. The temperature of the working fluid was kept approximately constant using a water cooler in order not to change the kinematic viscosity. The geometry of the IA and parent vessel model is displayed in Fig. 3. The actual aneurysm has a complicated 3D shape. However, in order to clarify the physical phenomenon with high generality and reproducibility, the IA and parent vessel model had a $2 \mathrm{D}$ projection shape with a $5 \mathrm{~mm}$ diameter straight parent vessel and $10 \mathrm{~mm}$ diameter sphere IA with constant thickness in this study. The thickness of the IA and parent vessel model was $5 \mathrm{~mm}$ in order not to change the hydraulic diameter $D_{\mathrm{H}}$. The IA and parent vessel model was made by machine processing acrylic resin, with the IA part and parent vessel part prepared separately and assembled later in order to accurately place the mesh sheet of the stent model. The straight section of the parent vessel model was set to over $500 \mathrm{~mm}$ upstream and over $200 \mathrm{~mm}$ downstream of the IA model to obtain fully developed laminar flow and to avoid flow disturbance at the end of the parent vessel model. The Reynolds number $(R e)$ of the working fluid flowing through the parent vessel model with square crosssection was defined as follows:

$\operatorname{Re}=\frac{\bar{U} D_{H}}{v}=\frac{Q}{D_{H} v}$

where $\bar{U}$ is the average flow velocity at the cross-section of the parent vessel and the $Q$ flow rate at the cross-section of

Table 1 Pore size (area of a pore) and porosity (aperture ratio of mesh area) of the fabricated stent models.

\begin{tabular}{ccc}
\hline & Pore size $\left[\mathrm{m}^{2}\right]$ & Porosity [\%] \\
\hline Bare Stent (BS) & $2.6 \times 10^{-6}$ & 84 \\
Flow Diverter (FD) & $3.9 \times 10^{-8}$ & 71 \\
Microporous Covered Stent (MCS) & $6.6 \times 10^{-9}$ & 36 \\
\hline
\end{tabular}

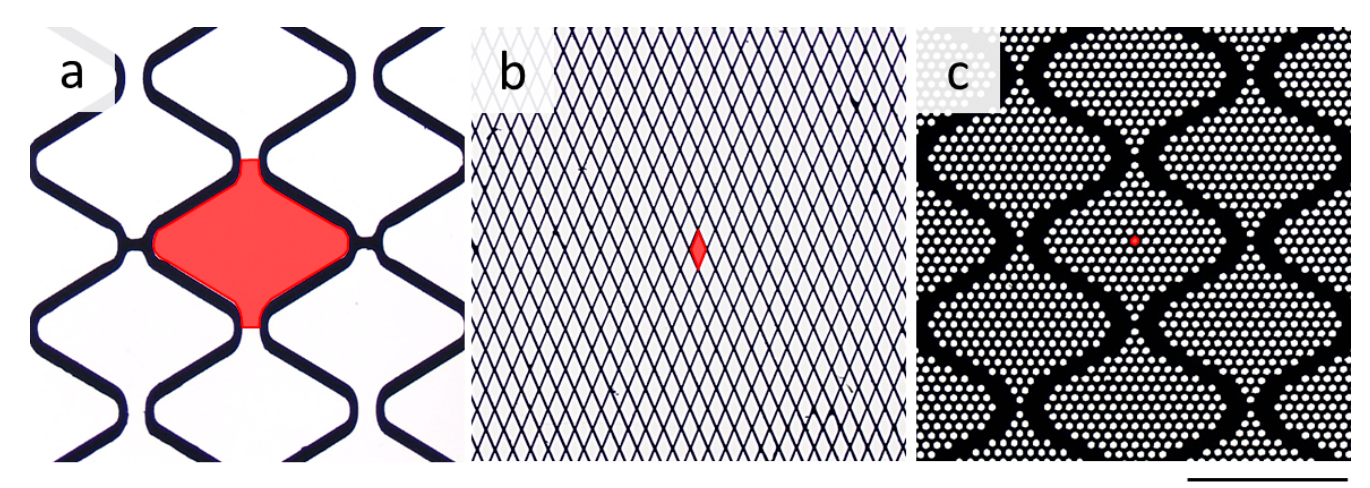

$2 \mathrm{~mm}$

Fig. 1 Optical micrograph of fabricated stent models: (a) bare stent (BS) model, (b) flow diverter (FD) model, (c) microporous covered stent (MCS) model. The red areas are the reference areas used for calculating pore size in Table 1. 


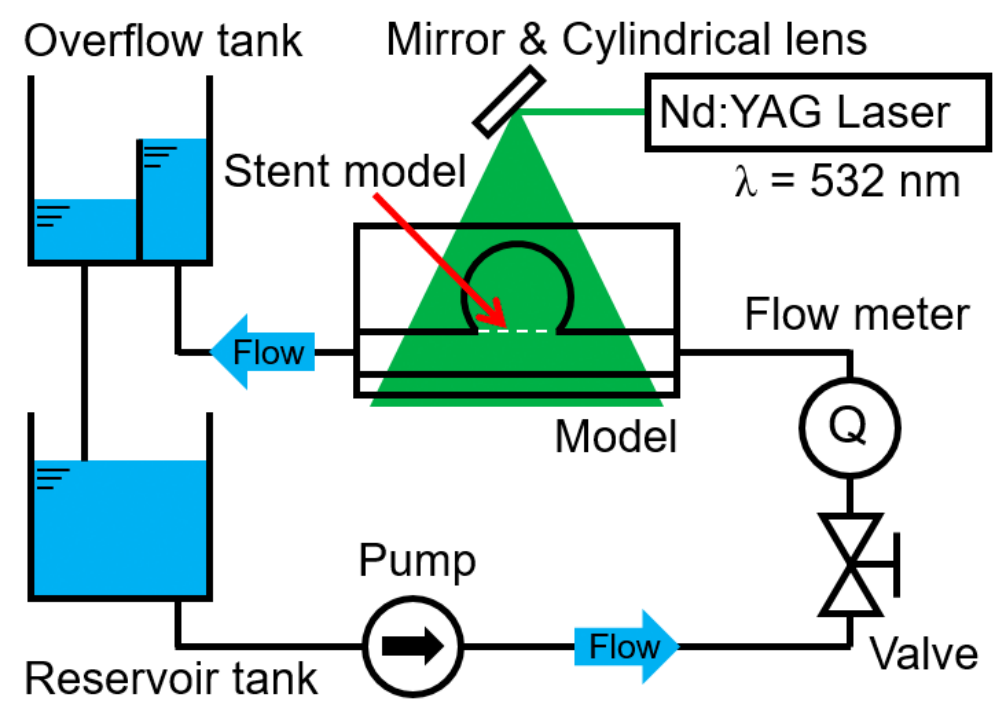

Fig. 2 Schematic diagram of in vitro flow simulator with IA model. The mesh sheet of the stent model was inserted between the IA and parent vessel model. PIV measurement was performed at the symmetry plane in the IA model.

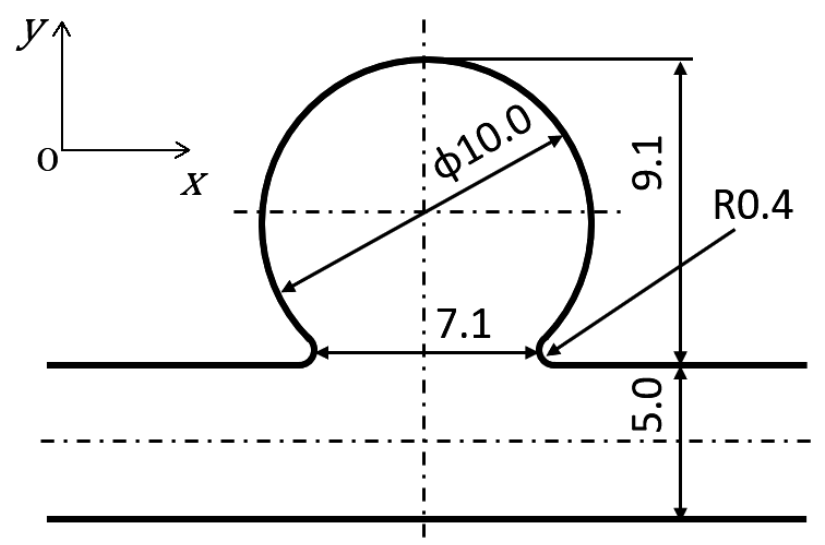

Fig. 3 Geometry of the IA and parent vessel model. The thickness of the IA and parent vessel is $5 \mathrm{~mm}$.

the parent vessel. $R e$ was varied in increments from 160 to 970 ( $Q: 210$ to $1280 \mathrm{ml} / \mathrm{min}$ ) by adjusting the flow rate using the flow regulating valve and the flow meter.

\subsection{PIV measurement}

In this study, the flow fields of the symmetry plane in the IA model were measured via the PIV technique. Hollow glass particles (density: $1100 \mathrm{~kg} / \mathrm{m}^{3}$, mean particle diameter: $0.01 \mathrm{~mm}$ ) were mixed with working fluid as tracer particles. The particles were illuminated using a $1 \mathrm{~mm}$ thick laser sheet generated by Nd:YAG Laser (DPIV-S50, LaVision $\mathrm{GmbH})$. The illuminated particles were captured using a CCD camera (Pixel fly QE-S Double shutter, PCO GmbH, 12 bit, $1392 \times 1024$ pixels) placed on the side of the IA model. The distributions of the velocity vector and shear rate of the IA flow were obtained using cross-correlation PIV (VidPIV 4.0, Intelligent Laser Applications GmbH) based on 100 timed images. The measurements were performed five times for each of the following four conditions: original (unstented condition), with BS, FD, and MCS models. Shear rate $\dot{\gamma}$ and area mean shear rate (AMSR) $\overline{\dot{\gamma}}$ were defined as follows.

$\dot{\gamma}=\frac{\partial v}{\partial x}+\frac{\partial u}{\partial y}$
$\overline{\dot{\gamma}}=\frac{\int_{A_{I A}}|\dot{\gamma}| d A}{\int_{A_{I A}} d A}$

where $v$ and $u$ are the flow speed in the $x$ and $y$-direction, and $A_{\mathrm{IA}}$ is the area of the projection shape of the IA model.

\section{Results}

\subsection{Velocity and shear rate map of IA flow}

As typical experimental results, the distributions of the flow velocity and shear rate for $R e \approx 600(Q \approx 790 \mathrm{ml} / \mathrm{min})$ are shown in Fig. 4. Before placing the stent model, fast swirling flow induced in the parent vessel flow was observed in the IA model. In addition, a high shear rate region was apparent in almost the whole area in the IA model. Following placement of the BS model, flow near the neck of the IA model was disturbed, and the flow velocity across the entire mapping area decreased. A high shear rate region was observed near part of the IA neck, but the shear rate at the depth of the IA was somewhat reduced. Following placement of the FD model, the direction of the swirling flow was opposite to that of the unstented condition and the BS model, and the flow velocity and shear rate decreased sig- 

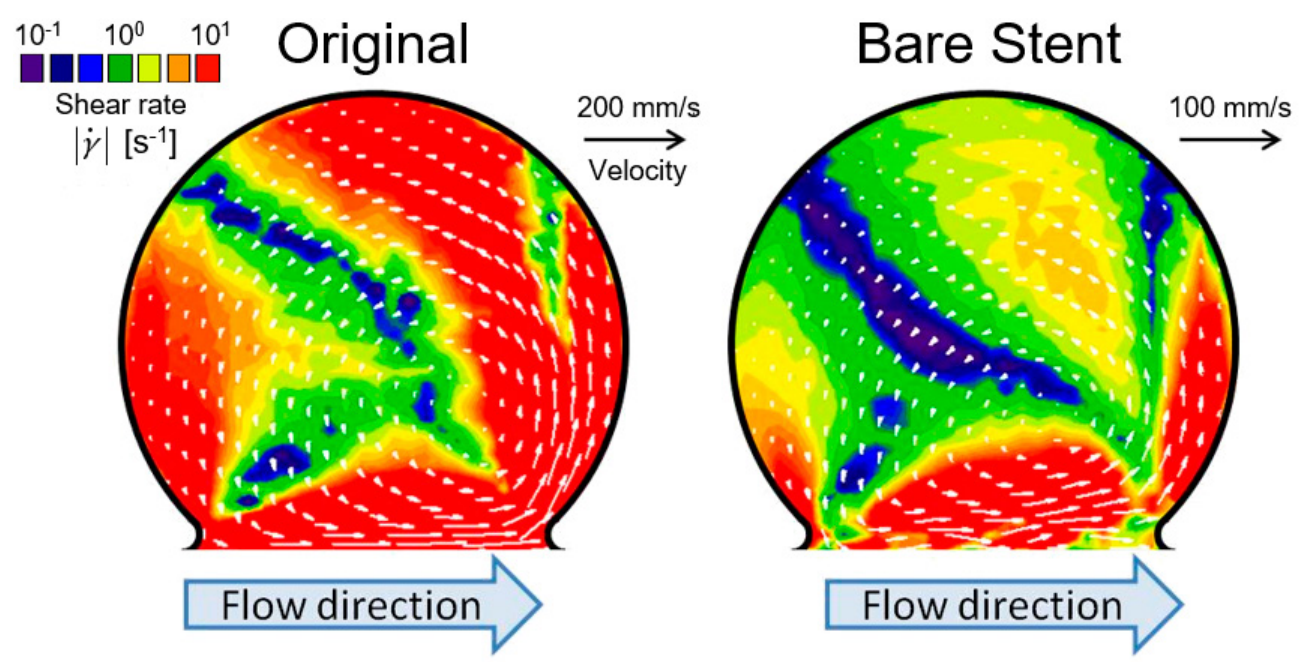

\section{Flow Diverter Microporous Covered Stent}
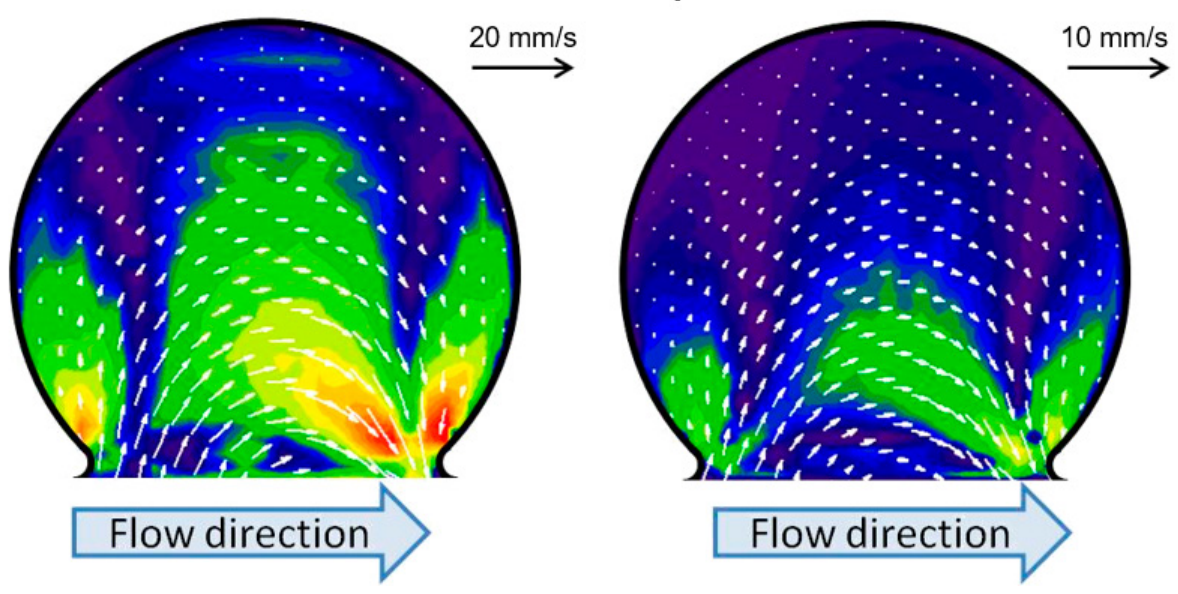

Fig. 4 Velocity (vector) and shear rate (color scale) in unstented (Original) and stented IA model. Shear rate is shown using common logarithmic scale.

nificantly. The flow velocity and shear rate reduced further when the MCS model was placed.

\subsection{Area mean shear rate}

In this paper, the AMSR of the whole mapping area in the IA model is used as the parameter for comparison of the flow reduction effect of the devices. Figure 5 shows the AMSR obtained in this PIV experiment. Following placement of any of the devices, the AMSR showed a correlation with the Reynolds number. At $R e \approx 600$, the AMSR was reduced to $34.7 \%\left(5.56 \mathrm{~s}^{-1}\right)$ for the BS model, $9.2 \%\left(1.48 \mathrm{~s}^{-1}\right)$ for the FD model, and $2.7 \%\left(0.43 \mathrm{~s}^{-1}\right)$ for the MCS model with respect to the unstented condition $\left(16.04 \mathrm{~s}^{-1}\right)$.

\section{Discussion}

In this study, the IA flow reduction property of MCS was measured and compared to that of FD. As described above, IA embolization stents promote intentional thrombosis in

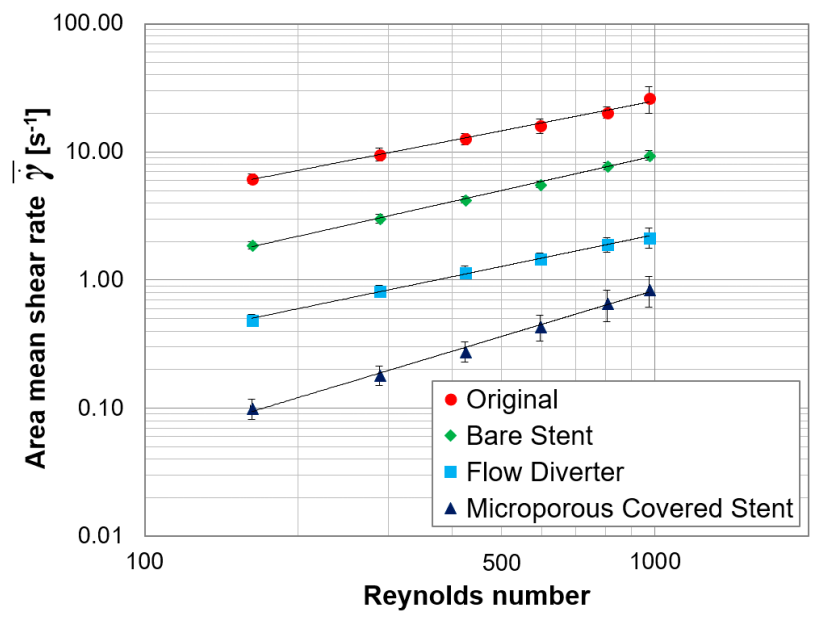

Fig. 5 The area mean shear rate in IA model with respect to $R e$ is shown in double logarithmic chart. Average value (plot) and standard deviation (bar) are shown $(n=5)$. Each line is an exponential fitted curve.

the IA by blood stagnation and facilitates IA embolization treatment by clotting of whole blood in the IA. In this scenario, 
a high-shielding stent is advantageous for IA embolization but is disadvantageous for side-branch vessel flow patency. The influence of blood flow shear rate on thrombosis promotion has been reported in previous papers [10, 11]; therefore, we only studied the relationship between the pore design of the shielding stent and the shear rate of IA flow [17]. Both reliable IA embolization and patency of the perforating arteries were achieved by placing MCS (NCVCCS1) in a previous study [9]. The main objectives of this study were evaluation of the flow reduction property of MCS's pore design and compare to that of FD, an existing IA embolization device, by focusing on the shear rate.

The transition of the flow vortex direction observed depends on the stent model, as shown in Fig. 4. In the unstented condition or with the BS model, the vortex flow is in the same direction as the parent vessel flow. The vortex flow is driven by the shear stress of the parent vessel flow to the fluid in the IA neck, and it is a relatively fast flow. By contrast, with the FD or MCS model, the vortex flow is in the opposite direction to that of the parent vessel flow. The vortex flow is driven by the pressure gradient at the IA neck, and it is a relatively slow flow with respect to the shear driven flow. The details of these vortex flows are presented in Ref. [17]; Bouillit et al. also described similar phenomena [15]. Placement of the FD or MCS model, with their strong flow reduction property, resulted in the shear stress of the parent vessel flow being sufficiently shielded and pressure gradient driven flow being observed in the IA model.

The AMSR of the IA flow at $R e \approx 600$ was one-third or less lower for the MCS model than for the FD model. From this result, it is clear that MCS's flow reduction property is superior to Pipeline Flex stent, and reliable IA embolization treatment can be provided by using MCS. Strong flow reduction can lead to occlusion of the side-branch where the stent is placed, but good patency was observed on the part of the MCS in previous study [9].

Several researchers $[15,16]$ have reported on the relationship between porosity and flow reduction property of stents. By contrast, Tajikawa et al. confirmed changes in the flow reduction property according to pore size, using stent models with almost the same porosity [17]. In this study, the AMSR of the IA flow at $R e \approx 600$ was one-third or less lower for the FD model than for the BS model. As the porosity of the FD model is not significantly different from that of the BS model, as shown in Table 1, it can be considered that difference in pore size also significantly affects the flow reduction property. The MCS model has smaller pores and lower porosity than the FD model, which results in its flow reduction property being significantly higher than that of the FD model.

In this study, the flow reduction property of the MCS model was compared with that of other stent models using a saccular IA model formed on the sidewall of a straight parent vessel. The results of the AMSR of IA flow are shown in Fig. 5. According to Kaibara, IA embolization may also occur in even BS. However, IA flow varies substantially depending not only on the parent vessel's flow rate but also the shape of the IA and the positional relationship between IA and the parent vessel. For example, the AMSR of wide-neck IA tends to be high as shear stress of the parent vessel's flow is easy to apply. In addition, at an IA formed on the outside-wall of the curved vessel, it is considered that the AMSR is very high because the parent vessel's flow directly enters the IA. In order to achieve reliable IA embolization at such an IA with fast flow, it is necessary to place a stent with strong flow reduction property. Differences in AMSR depending on aneurysm shape and/or parent vessel curvature will be investigated and reported in the near future.

\section{Conclusion}

In this paper, a hemodynamic study conducted using an in vitro flow simulator with a saccular IA model formed on the sidewall of a straight parent vessel model to compare the flow reduction property of our developed MCS with other stents was presented. The flow reduction property of the MCS was found to be approximately three times higher than that of the FD model. It is considerable that more reliable IA embolization than FD is obtained by using MCS, even for an IA with a fast flow, such as wide-neck IA or IA formed on the outside-wall of a curved vessel.

\section{Limitation}

PIV measurements were performed at the symmetry plane in the IA model, which had a 2D projection shape with suitable thickness in this study. However, the value of AMSR or the flow reduction percentage may differ in other planes, and so might the average characteristic of the 3D model. Furthermore, the flow reduction property in an in vitro experiment and the IA embolization effect in clinical and in vivo studies may not necessarily have a one-to-one relationship.

Acknowledgements This study was partly supported by a Grant-inAid for Young Scientists (B) (15K19985) from the Japan Society for the Promotion of Science, grants from the Ministry of Health, Labour and Welfare of Japan, and AMED(JP151k0103003).

\section{References}

1. Acciarri N, Toniato G, Raabe A, Lanzino G. Clipping techniques in cerebral aneurysm surgery. Journal of Neurosurgical Sciences. 2016; 60(1): 83-94.

2. Ernst M, Yoo AJ, Kriston L, Schönfeld MH, Vettorazzi E, Fiehler J. Is visual evaluation of aneurysm coiling a reliable study end point? Systematic review and meta-analysis. Stroke. 2015; 46(6): 1574-81. 
3. Hong Y, Wang YJ, Deng Z, Wu Q, Zhang JM. Stent-assisted coiling versus coiling in treatment of intracranial aneurysm: A systematic review and meta-analysis. PLOS One. 2014; 9(1): e82311.

4. Eller JL, Dumont TM, Sorkin GC, Mokin M, Levy EI, Snyder KV, Hopkins LN, Siddiqui AH. The pipeline embolization device for treatment of intracranial aneurysms. Expert Review of Medical Devices. 2014; 11(2): 137-50.

5. Wakhloo AK, Lylyk P, de Vries J, Taschner C, Lundquist J, Biondi A, Hartmann M, Szikora I, Pierot L, Sakai N, Imamura H, Sourour N, Rennie I, Skalei M, Beuing O, Bonafé A, Mery F, Turiman F, Brouwer P, Boccardi E, Valvassori L, Derakhshani S, Litzenberg MW, Gounis MJ. Surpass flow diverter in the treatment of intracranial aneurysms: A prospective multicenter study. AJNR American Journal of Neuroradiology. 2015; 36(1): 98-107.

6. Luecking H, Engelhorn T, Lang S, Goelitz P, Kloska S, Roessler K, Doerler A. FRED flow diverter: A study on safety and efficacy in a consecutive group of 50 patients. American Journal of Neuroradiology. 2017; 38(3): 596-602.

7. Nishi S, Nakayama Y, Ishibashi-Ueda H, Okamoto Y, Kinoshita Y. High-performance self-expanding stent graft: Development and application to experimental aneurysms. Journal of Artificial Organs. 2009; 12(1): 35-9.

8. Nishi S, Nakayama Y, Ishibashi-Ueda H, Masato Y. Occlusion of canine aneurysms using microporous self-expanding stent grafts: Long-term follow-up. Clinical Neurology and Neurosurgery. 2014; 122: 34-41.

9. Nakayama Y, Satow T, Funayama M, Moriwaki T, Tajikawa T, Furukoshi M, Hamano E, Ishi D, Hayashi M, Sugata S, IshibashiUeda H, Takahashi JC. Construction of 3 animal experimental models in the development of honeycomb microporous covered stents for the treatment of large wide-necked cerebral aneurysms.
Journal of Artificial Organs. 2016; 19(2): 179-87.

10. Yamane T, Maruyama O, Nishida M, Toyoda M, Tsutsui T, Jikuya T, Shigeta O, Sankai Y. The most profitable use of flow visualization in the elimination of thrombus from a monopivot magnetic suspension blood pump. Artificial Organs. 2004; 28(4): 390-7.

11. Kaibara M. Rheological study on coagulation of blood with special reference to the triggering mechanism of venous thrombus formation. Journal of Biorheology. 2009; 23(1): 2-10.

12. Bouillot P, Brina O, Ouared R, Lovblad KO, Farhat M, Pereira VM. Particle imaging velocimetry evaluation of intracranial stents in sidewall aneurysm: Hemodynamic transition related to the stent design. PLOS One. 2014; 9(12): e113762.

13. Gester K, Lüchtefeld I, Büsen M, Sonntag SJ, Linde T, Steinseifer $\mathrm{U}$, Cattaneo G. In vitro evaluation of intra-aneurysmal, flowdiverter-induced thrombus formation: A feasibility study. American Journal of Neuroradiology. 2016; 37(3): 490-6.

14. Morales HG, Bonnefous O, Geers AJ, Brina O, Pereira VM, Spelle L, Moret J, Larrabide I. Does arterial flow rate affect the assessment of flow-diverter stent performance? American Journal of Neuroradiology. 2016; 37(12): 2293-8.

15. Bouillot P, Brina O, Ouared R, Lovblad KO, Farhat M, Pereira VM. Hemodynamic transition driven by stent porosity in sidewall aneurysms. Journal of Biomechanics. 2015; 48(7): 1300-9.

16. Ohta M, Anzai H, Miura Y, Nakayama T. Parametric study of porous media as substitutes for flow-diverter stent. Biomaterials and Biomechanics in Bioengineering. 2015; 2(2): 111-25.

17. Tajikawa T, Nakagawa Y, Kurebayashi Y, Nishi S, Nakayama Y. Development of microporous covered stent for cerebral aneurysm treatment (Hydrodynamical evaluation of stent embolization and optimization of pore design of microporous film). Transactions of the Japan Society of Mechanical Engineers Series B. 2013; 79(801): 992-1004. (in Japanese) 